\title{
Equol Production Status is An Effective Predictor of Nonalcoholic Steatohepatitis in Women
}

\author{
Takemi Akahane ( $\nabla$ stakemi@naramed-u.ac.jp ) \\ Nara Medical University \\ Daisuke Kaya \\ Nara Medical University \\ Ryuichi Noguchi \\ Nara Medical University \\ Kosuke Kaji \\ Nara Medical University \\ Haruna Miyakawa \\ Saga Nutraceuticals Research Institute, Otsuka Pharmaceutical Co., Ltd \\ Yukihisa Fujinaga \\ Nara Medical University \\ Yuki Tsuji \\ Nara Medical University \\ Hiroaki Takaya
}

Nara Medical University

Yasuhiko Sawada

Nara Medical University

Masanori Furukawa

Nara Medical University

Koh Kitagawa

Nara Medical University

Takahiro Ozutsumi

Nara Medical University

Hideto Kawaratani

Nara Medical University

Kei Moriya

Nara Medical University

Tadashi Namisaki

Nara Medical University

Hitoshi Yoshiji

Nara Medical University 


\section{Research Article}

Keywords: Equol production status, effective predictor, nonalcoholic steatohepatitis, women, major soybean isoflavone, estrogenic and antioxidant activities.

Posted Date: June 14th, 2021

DOl: https://doi.org/10.21203/rs.3.rs-585963/v1

License: (c) (i) This work is licensed under a Creative Commons Attribution 4.0 International License.

Read Full License 


\section{Abstract}

Equol is a metabolite of daidzein, a major soybean isoflavone with estrogenic and antioxidant activities. As the production of equol depends on the presence of certain members of the intestinal microflora, not all individuals can produce equol. The incidence of nonalcoholic fatty liver disease (NAFLD) and nonalcoholic steatohepatitis (NASH) increases in menopausal women, and estrogen is associated with the progression of NAFLD/NASH. Therefore, the status of equol production might also be related to the pathogenesis of NAFLD/NASH in women. We, thus, examined the relationship between NASH histological features and equol production. Thirty-eight NAFLD patients who underwent liver biopsy were included in this study. In women, the degree of fibrosis and ballooning in nonproducers was significantly higher than that in producers. The percentage of nonproducers with NAFLD activity score (NAS) $\geq 5$ was significantly higher than that of producers. None of the histological features were significantly different between nonproducers and producers in men. Decision tree analysis identified the predictors of NAS $\geq 5$ in women. The status of equol production was the strongest predictor, followed by fasting glucose. Equol can be noninvasively detected in urine, suggesting its application as a screening test for predicting the diagnosis and progression of NASH in women.

\section{Introduction}

Nonalcoholic fatty liver disease (NAFLD) is globally the most common chronic liver disease, representing a significant health burden worldwide. Briefly, NAFLD includes a broad range of conditions, as among which simple steatosis and nonalcoholic steatohepatitis (NASH) ${ }^{1}$. The pathophysiology of NASH is multifactorial, involving genetic and epigenetic factors, insulin resistance, adipose-derived hormones, and nutritional factors ${ }^{2}$. The most potent driver of NASH is lipotoxicity-induced hepatocyte death, which triggers inflammation and fibrosis, leading to cirrhosis or liver cancer.

After menopause, women have an increased risk for insulin resistance, hyperlipidemia, and visceral fat accumulation, all of which are known risk factors for NAFLD ${ }^{3}$. A higher incidence of NAFLD is found in postmenopausal women than in premenopausal women, as estrogens inhibit stellate cell activation and fibrogenesis, suggesting their correlation with the progression of NAFLD/NASH ${ }^{4}$.

Soy-based foods, which are largely consumed in Asian countries, have become popular in non-Asian countries because of their beneficial effects on health. Equol, which is produced by the gut microbiota in the gastrointestinal tract, is a metabolite of daidzein, a major isoflavone found in soybean ${ }^{5}$. Equol has been reported to exert estrogenic activity, with affinity for both ER alpha and ER beta estrogen receptors. Equol is superior to all other isoflavones in its antioxidant activity ${ }^{6}$. As the production of equol depends on the presence of certain intestinal microflora, not all individuals have the ability to produce equol from the metabolism of isoflavones ${ }^{7}$. Only $25-30 \%$ of the adult population in Western countries have the ability to produce equol when fed soy-based foods containing isoflavones ${ }^{8-10}$. This percentage is significantly lower than the reported 50-60 \% frequency of equol-producers in adults from Japan, Korea, 
and China ${ }^{11-13}$, or Western adult vegetarians ${ }^{14}$. Usui et al. reported that the ratio of equol nonproducers in overweight or obese Japanese women was lower than the generally reported range ${ }^{15}$. Yoshikaka et al. reported that equol production was significantly associated with lower arterial stiffness and uric acid levels, and a high ratio of eicosapentaenoic acid to arachidonic acid in women in their $50 \mathrm{~s}^{16}$. As NAFLD/NASH has been associated with both the visceral fat area and metabolic syndrome, and the incidence of NAFLD/NASH is known to increase after menopause in women, the ability to produce equol is expected to be related to the pathogenesis of NAFLD/NASH; however, to date, no relationship has been reported between them.

Therefore, we aimed to examine the relationship between the pathogenesis of NASH and the status of equol production.

\section{Methods}

\section{Study populations}

Forty Japanese patients with clinically suspected NASH (13 men and 27 women) who visited the Department of Gastroenterology, Nara Medical University Hospital (Kashihara, Japan) between October 2017 and March 2019, were recruited for this study. All patients underwent a liver biopsy. The upper limit of alcohol consumption for NASH was defined as less than $30 \mathrm{~g} / \mathrm{d}$ for men and less than $20 \mathrm{~g} / \mathrm{d}$ for women in terms of ethanol content. Exclusion criteria included: those positive for hepatitis B surface antigen or antihepatitis $C$ virus antibodies, those with other hepatobiliary diseases, those on antibiotics or hormone therapy, those with diarrhea, and those unable to consume soy food products due to allergy or preference. Following the exclusion of 2 women who did not consume soy food products due to preference, a total of 38 (13 men and 25 women) patients were enrolled in this study.

\section{Clinical and Laboratory assessments}

The information collected using a questionnaire included alcohol consumption, medication history, and soy food consumption habits. In accordance with the definition of the Japanese Society of Obstetrics and Gynecology, women without a menstrual period for $₫ 12$ mo are considered menopausal. Hypertension was defined as a systolic blood pressure $\geq 140 \mathrm{mmHg}$, a diastolic blood pressure $\geq 90$ $\mathrm{mmHg}$, or current use of antihypertensive medication. Diabetes mellitus was defined as a fasting blood glucose level $\geq 126 \mathrm{mg} / \mathrm{dL}$ or current use of antidiabetic medication. Dyslipidemia was defined as the current use of lipid-lowering medication. Insulin resistance was assessed using the homeostasis model assessment-insulin resistance (HOMA-IR), which was calculated as fasting insulin $(\mu \mathrm{U} / \mathrm{mL}) \times$ fasting glucose $(\mathrm{mg} / \mathrm{dL}) / 405$. The FIB-4 index for noninvasive markers of liver fibrosis was calculated as follows: FIB-4 index $=($ age $[y] \times A S T) /\left(\right.$ platelet count $\left[10^{9} / \mathrm{L}\right] \times \sqrt{ }$ ALT $)$. The aspartate aminotransferase to platelet ratio index (APRI) for noninvasive markers of liver fibrosis was calculated as follows: [AST (IU/I) / AST (upper limit of normal IU/L) / platelet count $\left.\left(10^{9} / \mathrm{L}\right)\right] \times 100$.

\section{Analysis of urinary isoflavone and equol}


To determine the equal-producing status, participants consumed soy food products containing approximately $50 \mathrm{mg}$ isoflavones twice a day, and urine samples were collected the following morning. The levels of daidzein and equol in the urine of patients were measured in the Saga Nutraceuticals Research Institute (Otsuka Pharmaceutical Co., Ltd., Japan) by high-performance liquid chromatography (HPLC; Nexera X2; Shimadzu, Japan) using a type C18 column (Cortecs C18, $2.7 \mu \mathrm{m}, 3.0 \times 150$ mm; Waters, USA) and an SPD-M30A PDA and RF-20Axs detection system (Shimadzu), according to a modified method by Lundh et al. ${ }^{30}$. This method has been used for the detection of metabolites in human urine in previous studies ${ }^{31}$. Briefly, $100 \mu \mathrm{L}$ urine sample was deconjugated by incubating with 2 $\mu \mathrm{L}$ (ca. $300 \mathrm{U}$ ) $\beta$-glucuronidase (G0876, Sigma-Aldrich, USA) in sodium acetate buffer ( $\mathrm{pH} 5.0$ ) at $37^{\circ} \mathrm{C}$ for 30 min. Samples were then extracted using OASIS HLB microelution plates (Waters) prior to HPLC analysis. Quantitation was performed by means of UV response ( $254 \mathrm{~nm}$ and $280 \mathrm{~nm}$ ) for daidzein and fluorescence response (Ex: $255 \mathrm{~nm}, \mathrm{Em}: 310 \mathrm{~nm}$ ) for equol. The laboratory precision was assessed for each batch of samples through the analysis of the standard solution. The limits of quantification (LOQ) for daidzein and equol were 0.076 and $0.080 \mathrm{nmol} / \mathrm{mL}$, respectively. The equol-producing status of each urine collection was defined by using a log-transformed equol/daidzein ratio of -1.75 or more ${ }^{14}$.

\section{Pathology}

The liver tissue biopsy specimens were stained with hematoxylin-eosin, silver, and Azan stain, and analyzed by experienced pathologists blinded to the clinical data. NASH was defined as fat accumulation in more than $5 \%$ of hepatocytes, inflammation, and hepatocellular ballooning on liver biopsies. Disease activity was scored using the NAFLD activity score (NAS) of the Nonalcoholic Steatohepatitis Clinical Research Network. The scoring system comprised 4 histological features: steatosis (0-3), lobular inflammation (0-2), hepatocellular ballooning (0-2), and fibrosis (0-4) ${ }^{23,24}$. Accordingly, NAS is the unweighted sum of steatosis, lobular inflammation, and hepatocellular ballooning scores. The substages (1a, $1 \mathrm{~b}$, and $1 \mathrm{c})$ of fibrosis stage 1 were combined as a single stage.

\section{Statistical analysis}

All data were expressed as the mean \pm SD. Continuous variables were compared between 2 groups using the Student's $t$-test or the Mann-Whitney $U$ test, whereas categorical variables were compared using the chi-square test. Statistical significance was set at $p<0.05$. A decision-tree algorithm was constructed to reveal the profiles associated with an NAS $\geq 5$. The following factors were set as independent variables: age, menopause, hypertension, dyslipidemia, diabetes mellitus, BMI, platelet count, fasting glucose, HOMA-IR, ferritin, type 4 collagen 7S, P-III-P, FIB-4, APRI, and equol producers. All calculations were performed using the SPSS software (version 27, IBM Corp., Armonk, NY, USA).

\section{Results}

\section{Subject baseline characteristics}


We found that the number of equol nonproducers was 23 out of 38 . The prevalence of diabetes was shown to be significantly lower in nonproducers than in producers. We did not detect any significant differences in any of the other parameters tested between the 2 groups (Table 1). When subjects were stratified by sex, 7 male (53 \%) and 8 female (32\%) patients were identified as equol producers. No significant differences were detected in any of the tested parameters between nonproducers and producers in men. We observed, however, that in women the prevalence of diabetes was significantly lower, whereas the prevalence of dyslipidemia was higher, although not significantly different $(p=0.054)$, in nonproducers compared with those in producers (Table 2).

Table 1

Clinical characteristics of equol nonproducers and producers

\begin{tabular}{|c|c|c|c|}
\hline Variable & $\begin{array}{l}\text { Nonproducer } \\
(n=23)\end{array}$ & $\begin{array}{l}\text { Producer } \\
(n=15)\end{array}$ & $p$-Value \\
\hline Age, y & $54.6 \pm 15.0$ & $60.4 \pm 9.3$ & 0.697 \\
\hline Sex (Male/Female) & $(6 / 17)$ & $(7 / 8)$ & 0.191 \\
\hline Hypertension (\%) & $11(47.8)$ & $7(46.7)$ & 0.944 \\
\hline Dyslipidemia (\%) & $11(47.8)$ & $5(33.3)$ & 0.376 \\
\hline Diabetes mellitus (\%) & $11(47.8)$ & $12(80.0)$ & 0.047 \\
\hline $\mathrm{BMI}\left(\mathrm{kg} / \mathrm{m}^{2}\right)$ & $28.6 \pm 4.2$ & $28.5 \pm 4.1$ & 0.945 \\
\hline Platelet count $\left(\times 10^{\square} / \mu \mathrm{L}\right)$ & $20.3 \pm 6.4$ & $16.4 \pm 5.5$ & 0.062 \\
\hline AST (IU/L) & $62.0 \pm 26.7$ & $62.3 \pm 35.4$ & 0.970 \\
\hline ALT (IU/L) & $78.8 \pm 45.4$ & $73.0 \pm 41.2$ & 0.696 \\
\hline Fasting glucose (mg/dL) & $103.6 \pm 24.7$ & $115.7 \pm 30.0$ & 0.205 \\
\hline HOMA-IR & $5.8 \pm 5.8$ & $11.6 \pm 11.1$ & 0.129 \\
\hline Ferritin & $173.5 \pm 192.9$ & $238.0 \pm 128.0$ & 0.278 \\
\hline Type 4 collagen $7 \mathrm{~S}(\mathrm{ng} / \mathrm{mL})$ & $6.2 \pm 3.1$ & $6.0 \pm 2.2$ & 0.822 \\
\hline P-III-P (U/mL) & $0.7 \pm 0.3$ & $0.7 \pm 0.2$ & 0.895 \\
\hline FIB-4 index & $2.5 \pm 1.6$ & $3.3 \pm 2.7$ & 0.258 \\
\hline APRI & $1.1 \pm 0.7$ & $1.5 \pm 1.3$ & 0.214 \\
\hline
\end{tabular}

Table 2. Comparison of clinical characteristics between equol nonproducers and producers stratified by sex 


\begin{tabular}{|c|c|c|c|}
\hline Variable & $\begin{array}{l}\text { Nonproducer } \\
(\mathrm{n}=6)\end{array}$ & Producer $(\mathrm{n}=7)$ & $p$-Value \\
\hline Age, $y$ & $53.4 \pm 17.9$ & $56.0 \pm 8.4$ & 0.734 \\
\hline Hypertension (\%) & $3(50.0)$ & $4(57.1)$ & 0.797 \\
\hline Dyslipidemia (\%) & $2(33.3)$ & $4(57.1)$ & 0.391 \\
\hline Diabetes Mellitus (\%) & $4(66.7)$ & $5(71.4)$ & 0.853 \\
\hline $\mathrm{BMI}\left(\mathrm{kg} / \mathrm{m}^{2}\right)$ & $28.9 \pm 2.6$ & $28.5 \pm 3.7$ & 0.852 \\
\hline Platelet count $\left(\times 10^{4} / \mathrm{\mu L}\right)$ & $19.3 \pm 4.9$ & $17.6 \pm 5.0$ & 0.552 \\
\hline $\operatorname{AST}(\mathrm{IU} / \mathrm{L})$ & $60.2 \pm 16.3$ & $60.0 \pm 24.3$ & 0.989 \\
\hline ALT (IU/L) & $94.5 \pm 45.7$ & $78.4 \pm 45.6$ & 0.540 \\
\hline Fasting glucose $(\mathrm{mg} / \mathrm{dL})$ & $113.3 \pm 40.7$ & $129.9 \pm 27.8$ & 0.405 \\
\hline HOMA-IR & $9.4 \pm 9.8$ & $16.7 \pm 14.7$ & 0.397 \\
\hline Ferritin & $273.8 \pm 352.4$ & $242.6 \pm 140.0$ & 0.834 \\
\hline Type 4 collagen $7 \mathrm{~S}(\mathrm{ng} / \mathrm{mL})$ & $4.8 \pm 0.9$ & $5.6 \pm 2.9$ & 0.634 \\
\hline $\mathrm{P}-\mathrm{III}-\mathrm{P}(\mathrm{U} / \mathrm{mL})$ & $0.8 \pm 0.3$ & $0.7 \pm 0.2$ & 0.831 \\
\hline FIB-4 index & $1.9 \pm 1.0$ & $2.5 \pm 1.1$ & 0.414 \\
\hline APRI & $1.1 \pm 0.3$ & $1.3 \pm 0.6$ & 0.495 \\
\hline
\end{tabular}

\begin{tabular}{|c|c|c|c|}
\hline Variable & $\begin{array}{l}\text { Nonproducer } \\
(\mathrm{n}=17)\end{array}$ & Producer $(n=8)$ & $p$-Value \\
\hline Age, $y$ & $60.5 \pm 14.0$ & $64.1 \pm 8.8$ & 0.512 \\
\hline Hypertension (\%) & $8(47.1)$ & $3(37.5)$ & 0.653 \\
\hline Dyslipidemia (\%) & $9(52.9)$ & $1(12.5)$ & 0.054 \\
\hline Diabetes Mellitus (\%) & $7(41.2)$ & $7(87.5)$ & 0.030 \\
\hline Menopause (\%) & $14(82.4)$ & $7(87.5)$ & 0.743 \\
\hline $\mathrm{BMI}\left(\mathrm{kg} / \mathrm{m}^{2}\right)$ & $28.5 \pm 4.7$ & $28.5 \pm 4.7$ & 0.997 \\
\hline Platelet count $\left(\times 10^{4} / \mu \mathrm{L}\right)$ & $20.6 \pm 6.9$ & $15.4 \pm 6.0$ & 0.078 \\
\hline $\operatorname{AST}(\pi \mathrm{U} / \mathrm{L})$ & $62.6 \pm 30.1$ & $64.4 \pm 44.7$ & 0.907 \\
\hline $\operatorname{ALT}(\mathrm{IU} / \mathrm{L})$ & $73.2 \pm 45.3$ & $68.3 \pm 39.4$ & 0.797 \\
\hline Fasting glucose (mg/dL) & $100.1 \pm 16.5$ & $103.3 \pm 27.6$ & 0.725 \\
\hline HOMA-IR & $4.4 \pm 2.9$ & $8.6 \pm 8.2$ & 0.101 \\
\hline Ferritin & $144.0 \pm 117.2$ & $233.4 \pm 126.5$ & 0.111 \\
\hline Type 4 collagen $7 \mathrm{~S}(\mathrm{ng} / \mathrm{mL})$ & $6.5 \pm 3.3$ & $6.3 \pm 1.7$ & 0.853 \\
\hline P-III-P (U/mL) & $0.7 \pm 0.3$ & $0.7 \pm 0.2$ & 0.803 \\
\hline FIB-4 index & $2.7 \pm 1.8$ & $4.1 \pm 3.5$ & 0.317 \\
\hline APRI & $1.1 \pm 0.7$ & $1.7 \pm 1.7$ & 0.358 \\
\hline
\end{tabular}

Values are presented as $\mathrm{N}(\%)$ or mean \pm standard deviation. BMI, body mass index; AST, aspartate aminotransferase; ALT, alanine aminotransferase; HOMA-IR, homeostasis model assessment-insulin resistance; type III procollagen peptide; P-III-P, aspartate aminotransferase to platelet ratio index; APRI.

\section{Comparison of pathological features between equol nonproducers and producers}

We compared the 4 histological features of NAS (steatosis, lobular inflammation, hepatocellular ballooning, and fibrosis) between nonproducers and producers stratified by sex (Table 3 ). We noticed that in men, none of the histological features were significantly different between nonproducers and producers. In contrast, we found that in women, the degree of fibrosis in nonproducers was significantly higher than that in producers. Although we did not observe any stage 4 fibrosis in producers, the percentage of stage 4 fibrosis in nonproducers was found to be $23.5 \%$. Likewise, the degree of ballooning in nonproducers was shown to be significantly higher than that in producers, with the percentage of ballooning score 2 in nonproducers being $58.8 \%$, whereas that in producers being $12.5 \%$.

Table 3. Comparison of pathological features between equol nonproducers and producers stratified by sex

A. Male 


\begin{tabular}{|c|c|c|c|}
\hline & Nonproducer & Producer & $p$-Value \\
\hline Fibrosis stage & & & 0.292 \\
\hline 0 & 1, (16.7) & $0,(0)$ & \\
\hline 1 & $1,(16.7)$ & $0,(0)$ & \\
\hline 2 & $1,(16.7)$ & $1,(14.3)$ & \\
\hline 3 & $3,(50.0)$ & $3,(42.9)$ & \\
\hline 4 & $0,(0)$ & $3,(42.9)$ & \\
\hline Steatosis & & & 0.629 \\
\hline 1 & $0,(0)$ & $1,(14.3)$ & \\
\hline 2 & $5,(83.3)$ & $5,(71.4)$ & \\
\hline 3 & 1, (16.7) & $1,(14.3)$ & \\
\hline Lobular inflammation & & & 0.489 \\
\hline 1 & $2,(33.3)$ & $2,(28.6)$ & \\
\hline 2 & $3,(50.0)$ & $5,(71.4)$ & \\
\hline 3 & $1,(16.7)$ & $0,(0)$ & \\
\hline Ballooning & & & 0.612 \\
\hline 1 & $5,(83.3)$ & $5,(71.4)$ & \\
\hline 2 & 1, (16.7) & $2,(28.6)$ & \\
\hline NAS score & & & 0.292 \\
\hline 3 & $0,(0)$ & $1,(14.3)$ & \\
\hline 4 & $2,(33.3)$ & $0,(0)$ & \\
\hline 5 & $2,(33.3)$ & $4,(57.1)$ & \\
\hline 6 & $1,(16.7)$ & $2,(28.6)$ & \\
\hline 7 & $1,(16.7)$ & $0,(0)$ & \\
\hline
\end{tabular}

Values are $\mathrm{N}(\%)$.

B. Female 


\begin{tabular}{|c|c|c|c|}
\hline & Nonproducer $\mathrm{n},(\%)$ & Producer n, (\%) & $p$-Value \\
\hline Fibrosis stage & & & 0.047 \\
\hline 0 & $2,(11.8)$ & $0,(0)$ & \\
\hline 1 & $3,(17.6)$ & $0,(0)$ & \\
\hline 2 & $4,(23.5)$ & $7,(87.5)$ & \\
\hline 3 & $4,(23.5)$ & $1,(12.5)$ & \\
\hline 4 & $4,(23.5)$ & $0,(0)$ & \\
\hline Steatosis & & & 0.440 \\
\hline 1 & $6,(35.3)$ & $5,(62.5)$ & \\
\hline 2 & $7,(41.2)$ & $2,(25.0)$ & \\
\hline 3 & $4,(23.5)$ & $1,(12.5)$ & \\
\hline Lobular inflammation & & & 0.262 \\
\hline 0 & $1,(5.9)$ & $0,(0)$ & \\
\hline 1 & $5,(29.4)$ & $5,(62.5)$ & \\
\hline 2 & $11,(64.7)$ & $3,(37.5)$ & \\
\hline Ballooning & & & 0.030 \\
\hline 1 & $7,(41.2)$ & $7,(87.5)$ & \\
\hline 2 & $10,(58.8)$ & $1,(12.5)$ & \\
\hline NAS score & & & 0.084 \\
\hline 3 & $2,(11.8)$ & $3,(37.5)$ & \\
\hline 4 & $1,(5.9)$ & $3,(37.5)$ & \\
\hline 5 & $9,(52.9)$ & $1,(12.5)$ & \\
\hline 6 & $4,(23.5)$ & $1,(12.5)$ & \\
\hline 7 & $1,(5.9)$ & $0,(0)$ & \\
\hline
\end{tabular}

Values are $\mathrm{N}(\%)$.

We compared the percentage of nonproducers and producers with NAS $\geq 5$. We accordingly found that in men, the percentage of nonproducers with NAS $\geq 5$ was not significantly different from that in producers. However, in women, the percentage of nonproducers with NAS $\geq 5$ was demonstrated to be significantly higher than that in producers (Fig. 1).

\section{Prediction model for NAS $\geq \mathbf{5}$ using decision trees analysis}


To clarify the profiles associated with NAS $\geq 5$, we created a decision-tree algorithm. We set the following factors as independent variables: age, menopause, hypertension, dyslipidemia, diabetes mellitus, BMI, platelet count, fasting glucose, HOMA-IR, ferritin, type 4 collagen 7S, P-III-P, FIB-4, APRI, and equol producers. Among the factors expected to predict NAS $\geq 5$, we selected equol producers as the initial distinguishable factor for NAS $\geq 5$ in women. We observed a NAS $\geq 5$ in $25 \%$ of equol producers. In contrast, a NAS $\geq 5$ was identified in $82 \%$ of equol nonproducers. Fasting glucose was demonstrated to be the second most distinguishable factor among equol nonproducers. We observed a NAS $\geq 5$ in $93 \%$ of nonproducers with fasting glucose levels $>84 \mathrm{mg} / \mathrm{dL}$. In contrast, none of the nonproducer patients with fasting glucose $\leq 84 \mathrm{mg} / \mathrm{dL}$ was found to have a NAS $\geq 5$ (Fig. 2).

\section{Discussion}

We showed in this study that in women with $\mathrm{NASH}$, the degree of fibrosis and ballooning in equol nonproducers was significantly higher than that in producers. In addition, the percentage of nonproducers with NAS $\geq 5$ was significantly higher than that in producers in women with NASH. In contrast, in men with $\mathrm{NASH}$, no difference was identified between nonproducers and producers. As most women in this study were menopausal, it could be suggested that the status of equol production is associated with the pathogenesis of NASH in menopausal women.

The percentage of equol producers in Asia has been reported to be approximately $50-60 \%{ }^{12,17}$. In this study, $53 \%$ of men with NASH were equol producers, similar to a previous report ${ }^{17}$. However, in women with $\mathrm{NASH}$, the percentage of equol producers was $32 \%$, lower than previously reported ${ }^{12}$ and similar to the percentage of equol producers in obese women reported by Usui et al. ${ }^{15}$. Equol is produced from the isoflavone daidzein in the gut of humans and animals through the action of certain bacterial biotypes ${ }^{18}$. Gut dysbiosis has been significantly associated with obesity and the development and progression of NAFLD. The progression from NAFLD to NASH is known to largely occur due to bacterial dysbiosis, which activates inflammatory and profibrogenic intracellular pathways via Toll-like receptors (TLRs) and the activation of the inflammasome ${ }^{19}$. Bacterial dysbiosis might also be one of the causes of the inability of the host to produce equol, which in turn might be one of the reasons for the progression of NASH.

In women, the proportion of those with dyslipidemia was higher among equol nonproducers than producers, although the difference was not significant $(p=0.054)$. Some studies have reported a relationship between the level of serum lipids and the ability to produce equol. Yoshitaka et al. reported that equol producers had significantly lower visceral fat area, as well as lower levels of triglycerides and high-density lipoprotein (HDL) cholesterol compared with nonproducers ${ }^{16}$. Likewise, Guo et al. reported that among women equol producers had significantly lower levels of low-density lipoprotein (LDL) cholesterol than nonproducers ${ }^{20}$. Estrogen is known to increase the levels of HDL cholesterol and decrease those of LDL cholesterol, influencing body fat deposition. In addition, equol has been reported to exert the strongest estrogenic activity among all known isoflavones or isoflavone-derived metabolites 
21,22 . Both the central adiposity and levels of serum LDL cholesterol are known to increase in menopausal women. Therefore, the production of equol has been suggested to be associated with postmenopausal central adiposity and lipid metabolism.

In our study, the proportion of women affected with diabetes mellitus was higher in producers than nonproducers. Guo et al. reported that no significant difference was observed in the levels of fasting blood glucose between equol producers and nonproducers, neither in the overall group nor in patients stratified by sex ${ }^{20}$, suggesting that equol production probably had no effect on glucose metabolism. However, as diabetes is known to be closely related to the pathogenesis of $\mathrm{NASH}$, it is likely that diabetes has a greater impact on NASH in equol producers.

The diagnosis of NASH is established by the presence of a characteristic pattern of steatosis, inflammation, and hepatocellular ballooning on liver biopsies. Accordingly, a NAS threshold value (NAS $\geq$ 5 ) has been used for the diagnosis of $\mathrm{NASH}^{23,24}$. Our decision-tree analysis results showed that in women, the ability for equol production was the strongest predictor of NAS $\geq 5$, with fasting glucose being the second strongest predictor of NAS $\geq 5$ in equol nonproducers. As equol is thought to affect lipid metabolism and oxidative stress, a combination of the inability to produce equol and glucose metabolism abnormalities might increase the risk of NASH progression. As equol can be noninvasively measured in urine samples, it could be used as a useful screening tool for the prediction of NASH in women.

Equol is known to be superior to all other isoflavones in its antioxidant and estrogenic activities. Moreover, equol has been reported to be effective in improving the ischemic cardiovascular risk profile 25 , menopausal symptoms ${ }^{26,27}$, and suppression of decreased bone mineral density ${ }^{28,29}$. Although further research is needed, equol intake as a therapeutic strategy might lead to the improvement of NASH.

Our study had several limitations. The first limitation was the small sample size. Second, this study had a cross-sectional design that did not identify whether equol nonproducers progressed to NASH. Third, this was a single-center study.

\section{Conclusion}

Among women with NASH, equol nonproducers, which were also associated with fibrosis progression, had a significantly higher percentage of NAS $\geq 5$ than producers. The status of equol production was the strongest predictor of NASH in women. As equol can be noninvasively measured in urine, its detection could be employed as a simple screening test for predicting the diagnosis and progression of NASH in women.

\section{Declarations}

Ethics approval

Page $11 / 16$ 
This study was approved by the Ethics Committee of Nara Medical University (approval no. 1607). The study was conducted in concordance with the Helsinki Declaration. All included patients have signed an informed consent form prior to their participation in the study. All research was performed in accordance with relevant guidelines and regulations.

\section{Data availability}

The datasets generated during the current study are available from the corresponding author on reasonable request.

\section{Acknowledgements}

This work was supported by the Japan Society for the Promotion of Science (JSPS) KAKENHI (Grant Numbers 17K09439)

\section{Author contributions}

T.A. conceived the design, performed the research, and wrote the manuscript. D.K., R.N., and K.Ka. collected and analyzed the data. H.M. analyzed the data., Y.F., Y.T., H.T., Y.S., M.F., K.Ki., and T.O. collected the data. H. K., K.M., and T.N. edited the manuscript. H.Y. supervised the study. All authors reviewed the manuscript.

\section{Competing Interests Statement}

The authors declare no competing interests.

\section{References}

1. Younossi, Z. et al. Global Perspectives on Nonalcoholic Fatty Liver Disease and Nonalcoholic Steatohepatitis. Hepatology 69, 2672-2682, doi:10.1002/hep.30251 (2019).

2. Day, C. P. \& James, O. F. Steatohepatitis: a tale of two "hits"? Gastroenterology 114, 842-845, doi:10.1016/s0016-5085(98)70599-2 (1998).

3. Lovejoy, J. C., Champagne, C. M., de Jonge, L., Xie, H. \& Smith, S. R. Increased visceral fat and decreased energy expenditure during the menopausal transition. Int J Obes (Lond) 32, 949-958, doi:10.1038/ijo.2008.25 (2008).

4. Yang, J. D. et al. Gender and menopause impact severity of fibrosis among patients with nonalcoholic steatohepatitis. Hepatology 59, 1406-1414, doi:10.1002/hep.26761 (2014).

5. Xu, X., Harris, K. S., Wang, H. J., Murphy, P. A. \& Hendrich, S. Bioavailability of soybean isoflavones depends upon gut microflora in women. J Nutr 125, 2307-2315, doi:10.1093/jn/125.9.2307 (1995). 
6. Setchell, K. D., Brown, N. M. \& Lydeking-Olsen, E. The clinical importance of the metabolite equol-a clue to the effectiveness of soy and its isoflavones. J Nutr 132, 3577-3584, doi:10.1093/jn/132.12.3577 (2002).

7. Setchell, K. D. \& Clerici, C. Equol: history, chemistry, and formation. J Nutr 140, 1355s-1362s, doi:10.3945/jn.109.119776 (2010).

8. Lampe, J. W., Karr, S. C., Hutchins, A. M. \& Slavin, J. L. Urinary equol excretion with a soy challenge: influence of habitual diet. Proc Soc Exp Biol Med 217, 335-339, doi:10.3181/00379727-217-44241 (1998).

9. Atkinson, C., Frankenfeld, C. L. \& Lampe, J. W. Gut bacterial metabolism of the soy isoflavone daidzein: exploring the relevance to human health. Exp Biol Med (Maywood) 230, 155-170, doi:10.1177/153537020523000302 (2005).

10. Rowland, I. R., Wiseman, H., Sanders, T. A., Adlercreutz, H. \& Bowey, E. A. Interindividual variation in metabolism of soy isoflavones and lignans: influence of habitual diet on equol production by the gut microflora. Nutr Cancer 36, 27-32, doi:10.1207/s15327914nc3601_5 (2000).

11. Watanabe, S. et al. Pharmacokinetics of soybean isoflavones in plasma, urine and feces of men after ingestion of $60 \mathrm{~g}$ baked soybean powder (kinako). J Nutr 128, 1710-1715, doi:10.1093/jn/128.10.1710 (1998).

12. Arai, Y. et al. Comparison of isoflavones among dietary intake, plasma concentration and urinary excretion for accurate estimation of phytoestrogen intake. J Epidemio/ 10, 127-135, doi:10.2188/jea.10.127 (2000).

13. Song, K. B. et al. Prevalence of daidzein-metabolizing phenotypes differs between Caucasian and Korean American women and girls. J Nutr 136, 1347-1351, doi:10.1093/jn/136.5.1347 (2006).

14. Setchell, K. D. \& Cole, S. J. Method of defining equol-producer status and its frequency among vegetarians. J Nutr 136, 2188-2193, doi:10.1093/jn/136.8.2188 (2006).

15. Usui, T. et al. Effects of natural S-equol supplements on overweight or obesity and metabolic syndrome in the Japanese, based on sex and equol status. Clin Endocrinol (Oxf) 78, 365-372, doi:10.1111/j.1365-2265.2012.04400.x (2013).

16. Yoshikata, R., Myint, K. Z. \& Ohta, H. Relationship between equol producer status and metabolic parameters in 743 Japanese women: equol producer status is associated with antiatherosclerotic conditions in women around menopause and early postmenopause. Menopause 24, 216-224, doi:10.1097/gme.0000000000000743 (2017).

17. Akaza, H. et al. Comparisons of percent equol producers between prostate cancer patients and controls: case-controlled studies of isoflavones in Japanese, Korean and American residents. Jpn J Clin Oncol 34, 86-89, doi:10.1093/jjco/hyh015 (2004). 
18. Bolca, S. et al. Microbial and dietary factors are associated with the equol producer phenotype in healthy postmenopausal women. J Nutr 137, 2242-2246, doi:10.1093/jn/137.10.2242 (2007).

19. Pierantonelli, I. \& Svegliati-Baroni, G. Nonalcoholic Fatty Liver Disease: Basic Pathogenetic Mechanisms in the Progression From NAFLD to NASH. Transplantation 103, e1-e13, doi:10.1097/tp.0000000000002480 (2019).

20. Guo, K. et al. Daidzein-metabolising phenotypes in relation to serum lipids and uric acid in adults in Guangzhou, China. Br J Nutr 104, 118-124, doi:10.1017/s0007114510000279 (2010).

21. Setchell, K. D. et al. S-equol, a potent ligand for estrogen receptor beta, is the exclusive enantiomeric form of the soy isoflavone metabolite produced by human intestinal bacterial flora. Am J Clin Nutr 81, 1072-1079, doi:10.1093/ajcn/81.5.1072 (2005).

22. Jackson, R. L., Greiwe, J. S. \& Schwen, R. J. Emerging evidence of the health benefits of S-equol, an estrogen receptor $\beta$ agonist. Nutr Rev 69, 432-448, doi:10.1111/j.1753-4887.2011.00400.x (2011).

23. Brunt, E. M., Kleiner, D. E., Wilson, L. A., Belt, P. \& Neuschwander-Tetri, B. A. Nonalcoholic fatty liver disease (NAFLD) activity score and the histopathologic diagnosis in NAFLD: distinct clinicopathologic meanings. Hepatology 53, 810-820, doi:10.1002/hep.24127 (2011).

24. Kleiner, D. E. et al. Design and validation of a histological scoring system for nonalcoholic fatty liver disease. Hepatology 41, 1313-1321, doi:10.1002/hep.20701 (2005).

25. Liu, Z. M., Ho, S. C., Chen, Y. M., Liu, J. \& Woo, J. Cardiovascular risks in relation to daidzein metabolizing phenotypes among Chinese postmenopausal women. PLoS One 9, e87861, doi:10.1371/journal.pone.0087861 (2014).

26. Ishiwata, N., Melby, M. K., Mizuno, S. \& Watanabe, S. New equol supplement for relieving menopausal symptoms: randomized, placebo-controlled trial of Japanese women. Menopause 16, 141-148, doi:10.1097/gme.0b013e31818379fa (2009).

27. Aso, T. Equol improves menopausal symptoms in Japanese women. J Nutr 140, 1386s-1389s, doi:10.3945/jn.109.118307 (2010).

28. Ishimi, Y. Dietary equol and bone metabolism in postmenopausal Japanese women and osteoporotic mice. J Nutr 140, 1373s-1376s, doi:10.3945/jn.110.124842 (2010).

29. Weaver, C. M. \& Legette, L. L. Equol, via dietary sources or intestinal production, may ameliorate estrogen deficiency-induced bone loss. J Nutr 140, 1377s-1379s, doi:10.3945/jn.109.118331 (2010).

30. Lundh, T. J., Pettersson, H. \& Kiessling, K. H. Liquid chromatographic determination of the estrogens daidzein, formononetin, coumestrol, and equol in bovine blood plasma and urine. J Assoc Off Anal Chem 71, 938-941 (1988). 
31. Ideno, Y. et al. Optimal cut-off value for equol-producing status in women: The Japan Nurses' Health Study urinary isoflavone concentration survey. PLoS One 13, e0201318, doi:10.1371/journal.pone.0201318 (2018).

\section{Figures}
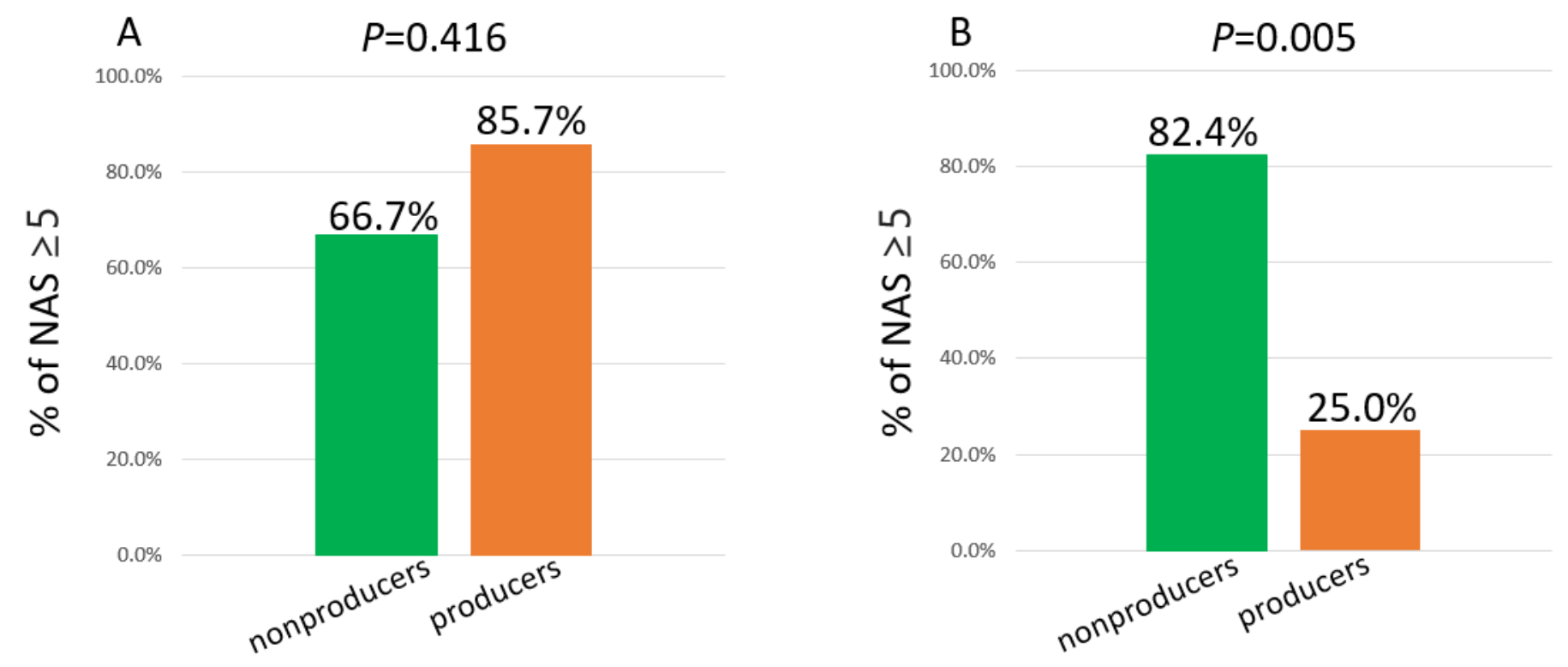

\section{Figure 1}

Comparison of the percentage of individuals with NAS $\geq 5$ between equol nonproducers and producers. A: Male. The percentage of nonproducers with NAS $\geq 5$ was not significantly different from that in producers. B: Female. The percentage of nonproducers with NAS $\geq 5$ was significantly higher than that in producers. 


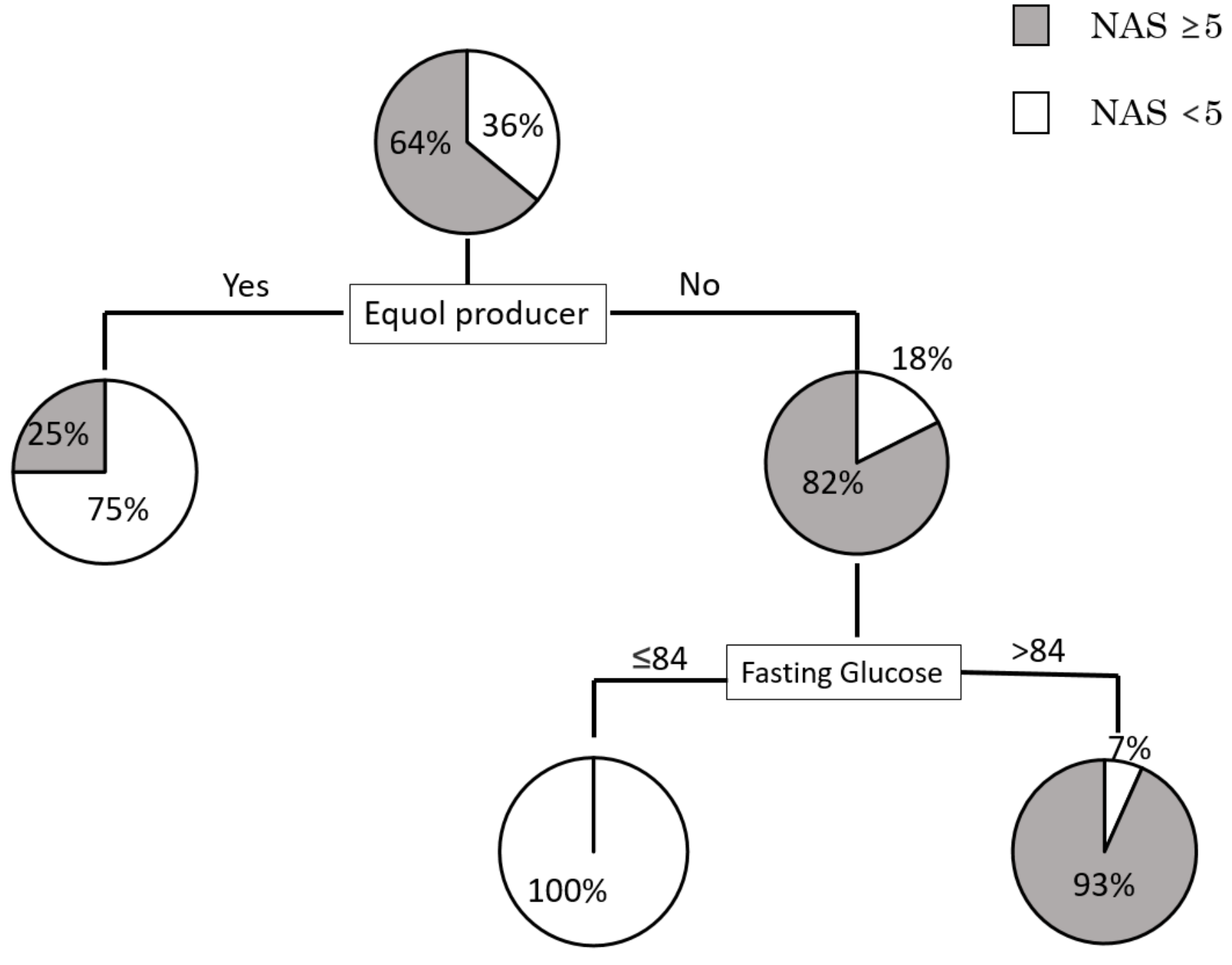

Figure 2

Decision tree analysis of factors predicting NAS $\geq 5$ in women. Pie graphs indicate the proportions of NAS $\geq 5$ (gray) and NAS $<5$ (white). 